\title{
Role of hsa-miR-325 in the etiopathology of preeclampsia
}

\author{
LEVENTE LÁZÁR, BÁLINT NAGY, ATTILA MOLVAREC, ANDRÁS SZARKA and JÁNOS RIGÓ Jr
}

First Department of Obstetrics and Gynecology, Semmelweis University Budapest, H-1085 Budapest, Hungary

Received February 2, 2012; Accepted June 8, 2012

DOI: $10.3892 / \mathrm{mmr} .2012 .954$

\begin{abstract}
Preeclampsia (PE) is a common pregnancy-specific syndrome characterized by hypertension and proteinuria. Evidence has demonstrated that hypertensive disorders in pregnancy are associated with alterations in the expression of different microRNAs (miRNAs). miRNAs are endogenously expressed non-coding RNAs that have significant biological and pathological functions due to their potential mechanisms of regulation of gene expression. The purpose of the present study was to investigate the expression of hsa-miR-325 in placental samples of preeclamptic and uncomplicated pregnancy patients. hsa-miR-325 was isolated from placenta tissue samples obtained from 31 preeclamptic and 28 normotensive pregnant females. Quantitative real-time polymerase chain reaction was used to analyze miRNA expression. The expression of hsa-miR-325 was elevated in uncomplicated pregnancies compared with preeclamptic patients. $\Delta \mathrm{Ct}$ (mean $\pm \mathrm{SD}$ ) values were $0.117 \pm 0.07$ in PE tissues and $0.135 \pm 0.051$ in normotensive cases $(\mathrm{p}<0.05)$. The expression levels correlated with patient blood pressure $(\mathrm{p}=0.015, \mathrm{r}=-0.23)$, and tended to correlate with body mass index $(\mathrm{p}=0.065, \mathrm{r}=0.261)$. The expression of hsa-miR-325 was downregulated in the case of PE. Changes in hsa-miR-325 expression in the case of pregnancy-related hypertensive disorders might affect the oxidative stress pathways and heat-shock protein production. These factors have a strong correlation with the development of PE. We, therefore, suggest that hsa-miR-325 contributes to the pathogenesis of PE.
\end{abstract}

\section{Introduction}

Hypertensive disorders are a leading cause of perinatal morbidity and mortality in pregnancy. Preeclampsia (PE) is a common pregnancy-specific syndrome that affects at least $5 \%$ of all pregnancies worldwide $(1,2)$. It is characterized by hypertension ( $R R \geq 140 / 90 \mathrm{mmHg}$ ) and proteinuria of $\geq 300 \mathrm{mg} / 24 \mathrm{~h}$, developing after midgestation in previously normotensive pregnant females. Although the exact etiology of PE remains unknown, pathological studies have

Correspondence to: Dr Levente Lázár, First Department of Obstetrics and Gynecology, Semmelweis University Budapest, 27 Baross Street, H-1085 Budapest, Hungary

E-mail: lazar_levente@hotmail.com

Key words: preeclampsia, hsa-miR-325, real-time PCR demonstrated the abnormal development of an ischemic placenta (2). Shallow endovascular trophoblast invasion in the spiral arteries and generalized endothelial cell dysfunction are key factors, while placental ischemia, oxidative stress and maternal-fetal immune maladaptation affect the development of PE. The only treatment is delivery of the placenta, after which the symptoms regress rapidly. Evidence has demonstrated that hypertensive disorders in non-pregnant and pregnant patients are associated with alterations in different miRNA expressions of specific tissues (3-5).

miRNAs are non-protein coding RNAs, and functionally negative regulators of gene expression by antisense complementarity to specific messenger RNAs (6,7). They act by targeting the RNA-induced silencing complex to complementary sites within the 3'-untranslated region (UTR) of their target mRNAs. Depending on the degree of base pairing between the miRNA and the 3'-UTR, either degradation or translational repression of the targeted mRNA occurs. Although they account for less than $1 \%$ of all human genes, miRNAs have been estimated to regulate up to $30 \%$ of all protein-encoding genes (8). Their best-known representatives are the 18-24 nucleotide long single-stranded miRNA. The mean number of copies per cell is between 10 and 50000 , depending on the tissue and the miRNA $(9,10)$.

Few studies have presented data regarding a comprehensive list of the human miRNAs expressed in the placenta and the possible roles of the different miRNAs in the pathophysiology of pregnancy-related disorders (11-13). Taking into consideration the high number of miRNAs, further studies regarding the expression of different miRNA in pregnancy-related hypertensive disorders may be important in understanding the pathophysiology of this disease.

In the present study, we performed a real-time polymerase chain reaction PCR analysis of hsa-miR-325. hsa-miR-325 is located at Xq21.1. Different databases (http://www.mirdb.org; http://www.nextprot.org) were evaluated, and hsa-miR-325 was selected as it is a non-studied miRNA that targets genes and candidate protein regulatory pathways affecting different etiological factors, including body mass index (BMI), blood pressure regulation, oxidative stress, endometrial function and heat-shock protein regulation, thus playing a key role in the development of hypertensive disorders in pregnancy.

\section{Materials and methods}

Samples. A total of 31 placenta samples were collected at delivery from females with PE and 28 from normotensive 
Table I. Clinical characteristics of normotensive and preeclamptic patients, and correlation with hsa-miR-325 expression in PE cases.

\begin{tabular}{lcccc}
\hline Variable & Controls (n=28) & Preeclampsia (n=32) & P-value & r-value \\
\hline Age (years) & $28(20-41)$ & $29(18-39)$ & NS & 0.003 \\
Systolic blood pressure (mmHg) & $110(90-135)$ & $160(138-210)$ & $<0.05$ & -0.13 \\
Diastolic blood pressure (mmHg) & $70(57-86)$ & $98(94-130)$ & $<0.05$ & -0.23 \\
Gestational age at sampling (weeks) & $36(35-40)$ & $37(30-41)$ & $\mathrm{NS}$ & 0.1 \\
Number of cesarean sections & $42 \%$ & $46 \%$ & $\mathrm{NS}$ & - \\
(\% of total deliveries in group) & & & $<0.05$ & 0.26 \\
BMI at delivery & $22.3(21.6-31.2)$ & $28.4(26.2-36.1)$ & $\mathrm{NS}$ & -0.01 \\
Fetal birth weight (grams) & $3600(2700-4200)$ & $3100(1800-3900)$ & & \\
\hline
\end{tabular}

r-value, correlation coefficient; PE, preeclampsia ; BMI, body mass index; NS, not significant.

pregnant females in this prospective study. The clinical characteristics of the patients are shown in Table I. All the females were pregnant with a single fetus. The tissue samples were collected $2-3 \mathrm{~cm}$ in radial distance from the margin of the placenta. Placenta samples were stored at $-80^{\circ} \mathrm{C}$. Small RNAs were obtained using the High Pure miRNA Isolation kit (Roche, Mannheim, Germany) according to the manufacturer's instructions. Strict anti-contamination procedures were used throughout. Double isolations were conducted from each placental sample. Each sample measurement was performed in triplicate.

miRNA detection. The miRNA profile of a certain tissue type is determined by miRNA microarray and sequencing. The small size of miRNAs makes the application of conventional quantitative PCR (QPCR) techniques difficult. However, this difficulty is relieved by using stem-loop or hairpin oligonucleotide QPCR. This method uses a primer with a hairpin structure during the reverse transcription which has a 5-8 base pair long overhang at the $3^{\prime}$ end specific to the miRNA, thus the size of cDNA transcribed from miRNA is increased during the transcription with the stem-loop region of the primer. The product generated through this method is suitable for quantitative examination with QPCR in the subsequent step. The benefits of the method are specificity (the reverse transcription and the quantitative PCR are miRNA specific) and sensitivity (the starting quantity of RNA is between 1 and $10 \mathrm{ng}$ ).

Specific detection and quantitative examination of miRNAs by stem-loop quantitative PCR was conducted with the use of Universal ProbeLibrary (UPL) technology (miRNA design software; UD-GenoMed Ltd.; Astrid Research Ltd.).

\section{Protocol}

Reverse transcription. The reverse transcription assay $(10 \mu \mathrm{l})$ comprised 10 ng RNA, 1 X reverse transcriptase buffer, 20 units RNase inhibitor, $1 \mathrm{mM}$ dNTP, 10 units reverse transcriptase and $50 \mathrm{nM}$ stem-loop primer. The components used for the reverse transcription were components of the Transcriptor First Strand cDNA Synthesis kit (Roche).

QPCR. The composition of the QPCR assay (10 $\mu \mathrm{l})$ was $2 \mu \mathrm{l}$ RT product, 1X LightCycler 480 Probes Master mix (Roche), $375 \mathrm{nM}$ forward and universal reverse primers
(Table II) and $125 \mathrm{nM}$ UPL probe. The quantitative PCR assay was conducted as follows: denaturation for $10 \mathrm{~min}$ at $95^{\circ} \mathrm{C}$, 45 cycles of $95^{\circ} \mathrm{C}$, annealing for $10 \mathrm{sec}$ at $58^{\circ} \mathrm{C}$ and $30 \mathrm{sec}$ at $72^{\circ} \mathrm{C}$, and extension for $10 \mathrm{~min}$ at $40^{\circ} \mathrm{C}$, which was conducted using the Applied LightCycler 480 Real-Time PCR system (Roche). The results were normalized using snoRNA202 RNA and the $\Delta \mathrm{Ct}$ method.

The threshold cycle of fluorescence $(\mathrm{Ct})$ for each sample was determined by real-time PCR in order to evaluate the association between the PE and the normotensive groups using the $\Delta \Delta \mathrm{Ct}$ method. $\Delta \Delta \mathrm{Ct}$ was the difference in the $\Delta \mathrm{Ct}$ value of $\mathrm{PE}$ and the mean $\Delta \mathrm{Ct}$ value of the normotensive cases $(\Delta \Delta \mathrm{Ct}=\Delta \mathrm{Ct} \mathrm{PE}-$ mean $\Delta \mathrm{Ct}$ normotensive $) . \Delta \mathrm{Ct}$ was the difference in the $\mathrm{Ct}$ value between the hsa-miR-325 and snoRNA202 $(\Delta C t=C t$ miR-325 - Ct snoRNA202). Differences in the expression levels of miRNA were measured by comparing the $\Delta \mathrm{Ct}$ values of the PE and normotensive groups.

The study protocol was approved by the Regional Institutional Committee of Medical Ethics at Semmelweis University, and written informed consent was obtained from each patient. The study was conducted in accordance with the Declaration of Helsinki.

Statistical analysis. Statistical analysis was determined using a paired t-test of the PE tissue samples compared to the normotensive cases. The association between miRNA expression levels and clinicopathological parameters was analyzed using a non-parametric test (Mann-Whitney U test between the two groups). $\mathrm{P}<0.05$ was considered to indicate a statistically significant difference. Statistical analysis was performed using the Statistical Program for Social Sciences (SPSS) software 16.0 (SPSS Incorporated, Chicago, IL, USA).

\section{Results}

Expression of hsa-miR-325. Expression levels of hsa-miR-325 were detected in $31 \mathrm{PE}$ and 28 normotensive placental tissue samples by real-time PCR. The values of $\Delta \mathrm{Ct}$ (mean $\pm \mathrm{SD}$ ) were $0.117 \pm 0.07$ in the PE tissues and $0.135 \pm 0.051$ in the normotensive cases (Fig. 1). Statistically significant differences were found in hsa-miR-325 and expression levels between the $\mathrm{PE}$ and normotensive placental tissues $(\mathrm{p}<0.05)$. A total of 
Table II. hsa-miR-325 primers.

\begin{tabular}{lc}
\hline Primer & Sequence \\
\hline Stem-loop RT & 5'-GTTGGCTCTGGTGCAGGGTCCGAGGTATTCGCACCAGAGCCAACACACTT-3' \\
Forward & 5'-GTCCTAGTAGGTGTCCAGT-3' \\
Universal reverse & 5'-GTGCAGGGTCCGAGGT-3'
\end{tabular}

A

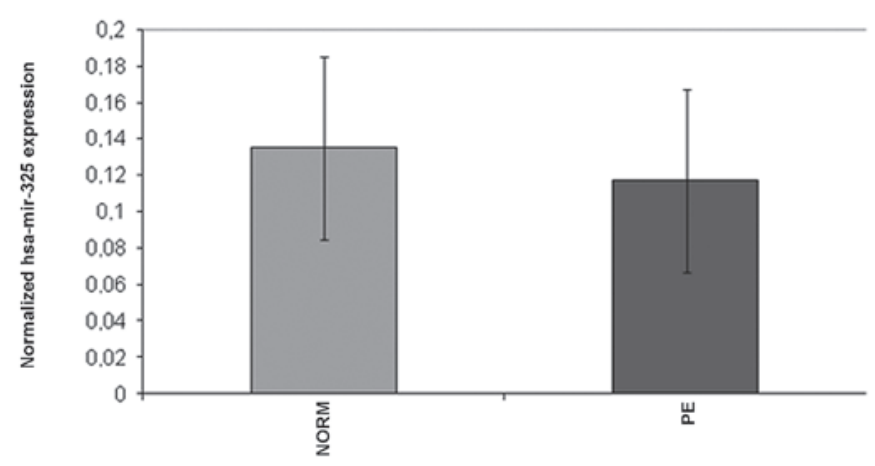

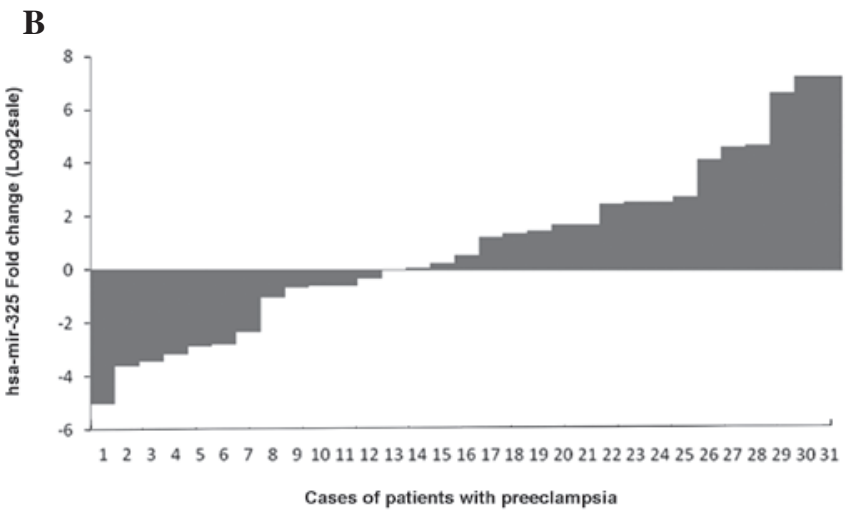

Figure 1. (A) Expression of hsa-miR-325 in 31 preeclamptic patients. Detection and quantitative examination of the miRNAs by stem-loop quantitative PCR with the use of UPL technology. Double isolations were conducted and each placental sample was then analyzed in triplicate. Data were presented as log2 of the fold-change of $\Delta \Delta \mathrm{Ct}(\Delta \Delta \mathrm{Ct}=\Delta \mathrm{Ct} \mathrm{PE}$ - mean $\Delta \mathrm{Ct}$ normotensive). (B) hsa-miR-325 was differentially expressed between PE and normotensive tissue samples. The miRNAs were normalized by snoRNA202. $\Delta \mathrm{Ct}=\mathrm{Ct}$ mir-325 - Ct snoRNA202. $\Delta \mathrm{Ct}$ values of hsa-mir-325 were significant in PE compared to normotensive patient tissue samples $(\mathrm{p}<0.05$, paired t-test).

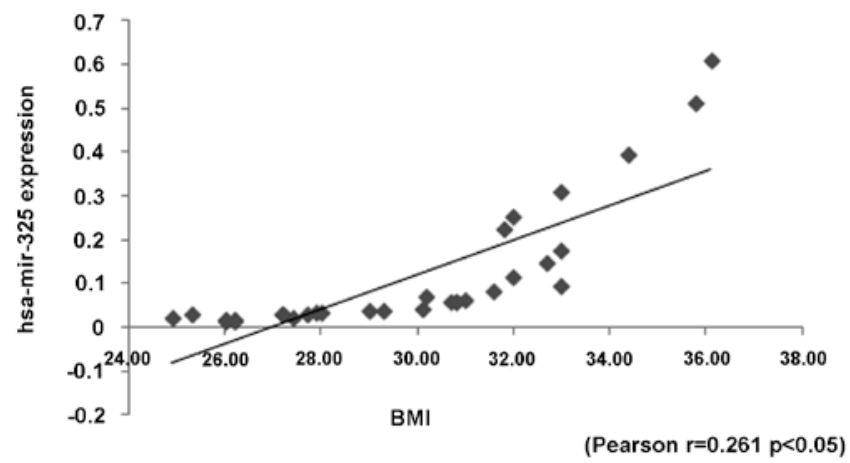

Figure 2. A strong correlation was found between hsa-miR-326 and BMI in PE cases. BMI, body mass index; PE, preeclampsia.

$70.9 \%(22 / 31)$ of the cases demonstrated a $>50 \%$ reduction in hsa-miR-325 expression levels in the PE tissues compared with the normotensive cases.

Association between the hsa-miR-325 expression levels and clinicopathological characteristics in PE. The downregulated hsa-miR-325 significantly correlated with diastolic blood pressure and BMI, respectively $(\mathrm{p}=0.015, \mathrm{p}=0.065$; Pearson correlation index: $-0.23,0.261$, respectively). Moreover, the expression levels of hsa-miR-325 were significantly lower in the patients with blood pressure $>160 / 110 \mathrm{mmHg}$ $(p=0.016)$. Therefore, the preeclamptic patients with severe PE had significantly lower expression levels of hsa-miR-325
(Fig. 2). However, there was no significant difference between hsa-miR-325 expression levels and other clinicopathological characteristics, including age, pregnancy age at delivery, method of delivery and fetal birth weight.

\section{Discussion}

$\mathrm{PE}$ is characterized by vasospasm, reduced placental perfusion and abnormal placentation. The main cause of fetal compromise is disturbance in uteroplacental perfusion. There are a number of hypotheses regarding the main cause of this disorder, including abnormal placentation, immunological background, and abnormal inflamatory response. We reviewed the literature with a focus on the etiological factors of PE and found that molecular pathways affecting etiological factors are important in the pathophysiology of PE. miRNAs have strong effects on the expression of different target genes and protein transcription.

Several studies have reported that specific miRNAs are overexpressed and underexpressed in PE (14). Pineles et al found that the expression of two miRNAs (miR-210 and miR-182) was significantly higher in PE compared to the control group (15). The absence or altered expression of these miRNAs may result in the re-programming of a number of their target genes in preeclamptic patients. Alteration of the miRNA expression in PE suggests the down- or upregulation of potential target genes which may contribute to the pathology of PE. The association between PE and the altered miRNA expression suggests the possibility of a functional role for miRNA in this disease. 
Our study presents an analysis of hsa-miR-325 expression in preeclamptic placental samples with the use of miRNA PCR technology. hsa-miR-325 is a less studied miRNA with possible target genes and proteins involved in the regulatory pathways of oxidative stress and heat shock protein regulation, including MAP4K4 (mediates TNF- $\alpha$ signaling and adipogenesis) serine/threonine kinase (plays a role in the response to environmental stress), CUGBP (Hsp70 regulation), UPF0554 [catalyzes the hydrolysis of ATP coupled with the exchange of $\mathrm{Na}(+)$ and $\mathrm{K}(+)$ ions across the plasma membrane, expressed in renal tubules and placental trophoblasts) and SLC25A13 (catalyzes the calcium-dependent exchange of cytoplasmic glutamate with mitochondrial aspartate across the mitochondrial inner membrane).

Our results indicate that hsa-miR-325 expression is associated with PE. Furthermore, we found a strong correlation between hsa-miR-325 and the blood presure of preeclamptic patients. miRNA expression also correlates with maternal BMI at delivery. Changes in hsa-miR-325 expression, in the case of pregnancy-related hypertensive disorders, may affect the oxidative stress pathways and heat-shock protein production. These factors have a strong correlation with the developement of PE (16-18). Our findings therefore may provide novel targets for further investigation of the pathogenesis of PE and these differential miRNAs may be potential markers for the diagnosis of PE. However, follow-up studies are required to confirm the significance of hsa-miR-325.

\section{Acknowledgements}

The study was designed by L.L., and performed by L.L. and B.N. Data were analyzed by L.L. and A.M. The manuscript was written by L.L. and B.N, with discussion suggestions by A.M., A.S. and J.R. This work was supported by the János Bolyai Research Scholarship of the Hungarian Academy of Sciences.

\section{References}

1. Witlin AG and Sibai BM: Hypertension in pregnancy: current concepts of preeclampsia. Annu Rev Med 48: 115-127, 1997.

2. Dekker GA and Sibai BM: Etiology and pathogenesis of preeclampsia: current concepts. Am J Obstet Gynecol 179: $1359-1375,1998$
3. Pineles BL, Romero R, Montenegro D, et al: Distinct subsets of microRNAs are expressed differentially in the human placentas of patients with preeclampsia. Am J Obstet Gynecol 196: 261, 2007.

4. Hu Y, Li P, Hao S, Liu L, et al: Differential expression of microRNAs in the placentae of Chinese patients with severe pre-eclampsia. Clin Chem Lab Med 47: 923-929, 2009.

5. Bátkai S and Thum T: MicroRNAs in hypertension: mechanisms and therapeutic targets. Curr Hypertens Rep 14: 79-87, 2012.

6. Bushati N and Cohen SM: MicroRNA functions. Annu Rev Cell Dev Biol 23: 175-205, 2007.

7. Griffiths-Jones S, Grocock RJ, Van Dongen S, et al: MiRBase: microRNA sequences, targets and gene nomenclature. Nucleic Acids Res 34: 140-144, 2006

8. Zhao S and Liu MF: Mechanisms of microRNA-mediated gene regulation. Sci China C Life Sci 52: 1111-1116, 2009.

9. Lim LP, Lau NC, Garrett-Engele P, et al: Microarray analysis shows that some microRNAs downregulate large numbers of target mRNAs. Nature 433: 769-773, 2005.

10. Draghici S, Khatri P, Martins RP, et al: Global functional profiling of gene expression. Genomics 81: 98-104, 2003.

11. Prieto DM and Markert ÚR: MicroRNAs in pregnancy. J Reprod Immunol 88: 106-111, 2011.

12. Maccani MA, Padbury JF and Marsit CJ: miR-16 and miR-21 expression in the placenta is associated with fetal growth. PLoS One 6: June 15,2011 (Epub ahead of print).

13. Kotlabova K, Doucha J and Hromadnikova I: Placental-specific microRNA in maternal circulation - identification of appropriate pregnancy-associated microRNAs with diagnostic potential. J Reprod Immunol 89: 185-191, 2011.

14. Zhu X-M, Han T, Sargent IL, et al: Differential expression profile of microRNAs in human placentas from preeclamptic pregnancies vs normal pregnancies. Am J Obstet Gynecol 200: 661, 2009.

15. Pineles BL, Romero R, Montenegro D, et al: Distinct subsets of microRNAs are expressed differentially in the human placentas of patients with preeclampsia. Am J Obstet Gynecol 3: 261-266, 2007.

16. Ekambaram P:HSP70 expression and its role in preeclamptic stress. Indian J Biochem Biophys 48: 243-255, 2011.

17. Asmathulla S, Koner BC and Papa D: Does oxidative stress play a role in altered plasma protein homeostasis in pregnancy-induced hypertension? Acta Physiol Hung. 98: 339-346, 2011.

18. Valenzuela FJ, Pérez-Sepúlveda A, Torres MJ, et al: Pathogenesis of preeclampsia: the genetic component. J Pregnancy 2012: 1 December, 2011 (E-pub ahead of print). 